\title{
THE RISING INCIDENCE OF TERMINAL 11Q DELETION?
}

\author{
R.K. Lall ${ }^{1}$, J. Brent ${ }^{1}$, C. Oley ${ }^{2}$, M. Ahmed ${ }^{1}$ \\ ${ }^{1}$ Paediatrics, Queen's Hospital Burton, Burton upon Trent, ${ }^{2}$ Cytogenetics, Birmingham Women's NHS \\ Foundation Trust, Birmingham, UK
}

Background and aim: First described in 1973, Jacobsen syndrome is a MCA/MR contiguous gene syndrome caused by partial deletion of the long arm of chromosome 11 with a prevalence of 1/100 000 births. Only 200 cases have been reported to date. There is a wide spectrum of severity of clinical phenotype; facial dysmorphism, multi organ involvement including haematological dysfunction with thrombocytopenia and clotting abnormalities, cardiac defects like hypoplastic heart, and renal anomalies. The presentation depends on the deletion size (5-16MB). There is also a high recurrence risk when a parent has a balanced translocation or deletion 11q in the mosaic form.

We share 2 cases of terminal 11q deletion diagnosed in the last 4 months in 1 district general hospital in West Midlands, UK and emphasize the growing need to be aware of this recent genetic condition.

Methods and results: Case 1 presented as Small for gestation (SGA) with thrombocytopenia but no obvious dysmorphic features.

Case 2 presented as SGA with facial dysmorphism and thrombocytopenia.

Our regional genetics laboratory identified 6 individuals with terminal $11 \mathrm{q}$ deletions in the west midlands in the last 2 years.

Conclusion: Half of patients with terminal 11q deletion are diagnosed by 1 year of age and the rest even later. Not all patients present with typical phenotypic features and could easily escape neonatal diagnosis where early detection could improve management of potentially life threatening complications. Clinicians should therefore be more aware of this condition which may only present with thrombocytopenia and SGA. 